Hardy-Ramanujan Journal

Vol.12 (1989) 14-19

\title{
A TRIVIAL REMARK ON GOLDBACH CONJECTURE \\ BY
}

K. RAMACHANDRA

§ 1. INTRODUCTION. In [3] S. Srinivasan has proved the following conditional theorem. (We write $e(x)=\exp (2 \pi i x)$ ).

THEOREM. Let $B>10$ be arbitrary. Suppose there is a function $\delta_{B}$ tending to zero as $B \rightarrow \infty$, such that

$$
\left|\int_{M_{3}} S^{2}(\alpha) e(-N \alpha) d \alpha\right|<\delta_{B} \frac{N}{(\log N)^{2}}\left(\frac{N}{\varphi(N)}\right),
$$

where $S(\alpha)=\sum_{p \leq X} e(p \alpha)(p:$ primes $), N=[X]$ and $\mathcal{M}_{3}$ is the part of the minor arcs corresponding to denominators $q$,

$$
Q(\log X)^{-2}<q<Q
$$

with $Q=X(\log X)^{-B}$. Then the number $r(N)$ of representations of $N$ as a sum of two odd primes satisfies

$$
r(N)=(1+o(1)) S_{0}(N) T(N)
$$


as $N \rightarrow \infty$, where $T(N)=\sum\left(\log m_{1} \log m_{2}\right)^{-1}$ the summation being over integers $m_{1}>1, m_{2}>1$ and $m_{1}+m_{2}=N$, and

$$
S_{0}(N)=\prod_{p \mid N}\left(1-\frac{1}{(p-1)^{2}}\right) \prod_{p \mid N}\left(1+\frac{1}{p-1}\right)
$$

The analogous statement holds good for the twin-primes conjecture also.

In this note we prove the theorem above with $Q(\log X)^{-2}<q<Q$ replaced by $Q(\log X)^{-1-\varepsilon}<q<Q$ where $\varepsilon>0$ is any small constant. (In conversation with Srinivasan I came to know that he also knew this result. In fact it follows by his conditions on $A, B, C$. However my treatment is different.)

We now describe his basic and supplementary intervals. Let

$$
L=\log X, Q=X L^{-B}, Q_{1}=L^{A}, Q_{0}=X L^{-(B+C)}
$$

where the constants $A, B, C$ satisfy

$$
A>3(B+C), B>1, C>1 .
$$

Let $\mathcal{M}_{1}$, be the union of $\alpha$-intervals defined by

$$
\left|\alpha-\frac{h}{q}\right|<\frac{1}{Q}, 0 \leq h<q,(h, q)=1, \text { and } q \leq Q_{1} .
$$

These intervals are disjoint and

$$
\int_{\mathcal{M}_{1}} S^{2}(\alpha) e(-N \alpha) d \alpha=T(N) S_{0}(N)+O\left(\frac{1}{A} \frac{N}{Q(N)} \frac{N}{(\log N)^{2}}\right)
$$

$\mathcal{M}_{2}$ is in union of the $\alpha$-intervals defined by

$$
M_{2}=\bigcup_{Q_{1} \leq q \leq Q_{0}} \bigcup_{\substack{k=0 \\(h, q)=1}}^{q-1} \mathcal{M}(h, q)
$$

where $\mathcal{M}(h q)=\left[\frac{h}{q}-(q Q)^{-1}, \frac{h}{q}+(q Q)^{-1}\right]$. 
Let

$$
I=\left[-Q^{-1}, 1-Q^{-1}\right] \text { and } \mathcal{M}=\bigcup_{1 \leq q \leq Q} \bigcup_{\substack{h=0 \\(h, q)=1}}^{q-1} \mathcal{M}(h, q)
$$

We know that $M=I$ and we have to get an asymptotic formula for

$$
\int_{I} S^{2}(\alpha) e(-N \alpha) d \alpha
$$

We put $M_{3}=I-\left(M_{1} \cup M_{2}\right)$ and we get the result of Srinivasan. (To sum up, the basic iniervais treatment of Ŝrinivasan follows Prachar (see [2] p.182) and that of Gallagher (see [1], Lemma 7) and his supplementary intervals treatment involves a simple but new idea). We give in the next section our new treatment of supplementary intervals.

§ 2. NEW TREATMENT OF SUPPLEMENTARY INTERVALS. In Lemmas 2 to 5 constants implied by the Vinogradov symbol $\ll$ depend on the integer constant $k$.

LEMMA 1. Let $v$ and $\gamma$ be any two real numbers and $f(x)$ a $k$ times continuously differentiable function defined in $v \leq x \leq v+k \gamma$. Then

$$
\begin{gathered}
\int_{0}^{\gamma} \cdots \int_{0}^{\gamma} f^{k}\left(v+u_{1}+\cdots+u_{k}\right) d u_{1} \cdots d u_{k} \\
=f(v+k \gamma)-\left(\begin{array}{l}
k \\
1
\end{array}\right) f(v+(k-1) \gamma)+-\cdots+(-1)^{k} f(v) .
\end{gathered}
$$

PROOF. The lemma follows by induction on $k$.

LEMMA 2. Let $Q_{0}, Q_{1}, Q$ and $q$ be positive integers with

$$
Q_{1} \leq q \leq Q_{0} \leq Q
$$

Let $a$ be an integer satisfying $1 \leq a \leq q,(a, q)=1, \gamma$ a real number satisfying $|\gamma| \leq \frac{1}{q Q_{0}}, k$ a fixed natural number, $v=\frac{a}{q}+\beta$ with $0 \leq|\beta| \leq \frac{1}{q Q}$. Put $F(x)=\sum_{p \leq N} e(p x)$ where $N$ is any natural number and $f(x)=|F(x)|^{2}$.

Then

$$
f(v) \ll \frac{N^{k+2}}{(\log N)^{2}\left(q Q_{0}\right)^{k}}+\sum_{j=1}^{k} f(v+j \gamma)
$$


PROOF. The first term on the RHS comes from a trivial estimation of the multiple integral in Lemma 1 and the second term is obvious.

LEMMA 3. We have,

$$
\begin{gathered}
\frac{2 f(v)}{q Q_{0}}=\int_{|\gamma| \leq \frac{1}{Q_{0}}} f(v) d \gamma \\
\ll \frac{N^{k+2}}{(\log N)^{2}\left(q Q_{0}\right)^{k+1}}+\int_{|w| \leq \frac{k+1}{Q_{0}}} f\left(\frac{a}{q}+w\right) d w .
\end{gathered}
$$

PROOF. Follows from Lemma 2 on integration with respect to $\gamma$ since for $1 \leq j \leq k$ we have

$$
\int_{|\gamma| \leq \frac{1}{Q_{0}}} f\left(\frac{a}{q}+\beta+j \gamma\right) d \gamma \ll \int_{|w| \leq \frac{k+1}{Q_{0}}} f\left(\frac{a}{q}+w\right) d w
$$

(on putting $\beta+j \gamma=w$ ).

LEMMA 4. Any fixed interval

$$
\frac{a}{q} \pm \frac{k+1}{q Q_{0}}\left(1 \leq a \leq q,(a, q)=1,1 \leq q \leq Q_{0}\right)
$$

intersects at most $O(1)$ other intervals and so

$$
\begin{aligned}
& \sum_{q \leq Q_{0}} \sum_{a} \int_{|\beta| \leq \frac{1}{Q}} \int_{|w| \leq \frac{k+1}{Q}} f\left(\frac{a}{q}+w\right) d w \\
& \ll\left(\int_{0}^{1} f(x) d x\right) \frac{Q_{0}}{Q} \ll \frac{N}{\log N}\left(\frac{Q_{0}}{Q}\right) .
\end{aligned}
$$

PROOF. Follows by the remark that the innermost integral on the LHS is independent of $\beta$.

LEMMA 5. We have

$$
\sum_{Q_{1} \leq q \leq Q_{0}} \sum_{a} \int_{|\beta| \leq \frac{1}{q}} f\left(\frac{a}{q}+\beta\right) d \beta
$$




$$
\ll \frac{N^{2}}{Q(\log N)^{2}}\left(\frac{N}{Q_{0} Q_{1}}\right)^{k}+\frac{N}{\log N}\left(\frac{Q_{0}}{Q}\right)
$$

PROOF. By Lemma 3, we have,

$$
\sum_{a} f\left(\frac{a}{q}+\beta\right)<\frac{N^{k+2} q Q_{0}}{(\log N)^{2}\left(q Q_{0}\right)^{k+1}}+q Q_{0} \sum_{a} \int_{|\gamma| \leq \frac{k+1}{Q_{0}}} f\left(\frac{a}{q}+w\right) d w .
$$

Integrating with respect to $\beta$ in $|\beta| \leq \frac{1}{q Q}$ and summing over $q$ in

$$
Q_{1} \leq q \leq Q_{0}
$$

we obtain

$$
\begin{gathered}
\sum_{Q_{1} \leq q \leq Q_{0}} \sum_{a} \int_{|\beta| \leq \frac{1}{q}} f\left(\frac{a}{q}+\beta\right) d \beta \\
\frac{N^{k+2}}{(\log N)^{2}} \sum_{q \geq Q_{1}} \frac{q Q_{0}}{\left(q Q_{0}\right)^{k+1}}\left(\frac{1}{q Q}\right)+\left(\frac{Q_{0}}{Q}\right)\left(\frac{N}{\log N}\right) \\
\ll \frac{N^{2}}{Q(\log N)^{2}}\left(\frac{N}{Q_{0} Q_{1}}\right)^{k}+\left(\frac{Q_{0}}{Q}\right)\left(\frac{N}{\log N}\right) .
\end{gathered}
$$

This proves the lemma.

§ 3. REMARKS. The basic intervals are already investigated by S. Srinivasan [3]. The application of the results of $\$ 2$ to estimation of a trivial portion of supplementaryintervals is clear. For instance we can take

$$
Q=\frac{N}{(\log N)^{A}}, Q_{0}=N(\log N)^{-A-1-\varepsilon}, Q_{1}=(\log N)^{A+1+2 \varepsilon}, k \geq k_{0}(\varepsilon, A)
$$

ACKNOWLEDGEMENT. The author wishes to thank Dr. S. SRINIVASAN for going through an earlier draft of this note. 


\section{REFERENCES}

1. P.X. GALLAGHER, Primes and powers of 2, Invent Math. 29 (1975), 125-142.

2. K. PRACHAR, Primzahlverteilung, Berlin/New York, (1957).

3. S. SRINIVASAN, A remark on Goldbach problem, J. Number Theory 12 (1980), 116-121.

\section{ADDRESS OF THE AUTHOR}

PROFESSOR K. RAMACHANDRA

SCHOOL OF MATHEMATICS

TATA INSTITUTE OF FUNDAMENTAL RESEARCH

HOMI BHABHA ROAD

BOMBAY 400005

INDIA 